\title{
MOVILIZACIÓN DEL CONOCIMIENTO EN EDUCACIÓN. CONEXIÓN ENTRE LA INVESTIGACIÓN, LA POLÍTICA Y LA PRÁCTICA: UNA APROXIMACIÓN TEÓRICA
}

Knowledge mobilization in education. Connection between the research, the

\author{
policy and practice: a theoretical approach
}

http://dx.doi.org/10.22235/pe.v10i1.1362

\section{HAYLEN PERINES ${ }^{1}$}

Universidad Autónoma de Madrid. España.

Correspondencia: Haylen Perines, Universidad Autónoma de Madrid.

Recibido: 14-09-2016

Correo electrónico: profesorahaylen@gmail.com

Revisado: 25-09-2016

Aceptado: 30-09-2016

Resumen: Este artículo analiza el concepto de knowledge mobilization (movilización del conocimiento en español) desde su importancia como término novedoso en el contexto educativo contemporáneo. Las formas en que los conocimientos son transmitidos a la comunidad educativa forjaron una creciente línea de investigación que analiza cómo se movilizan estos saberes desde las universidades y centros de investigación hacia los agentes educativos y a la sociedad. A través de una exhaustiva revisión teórica, este trabajo presenta los fundamentos del concepto, describe estudios empíricos que lo abordan y menciona las sugerencias que realizan los estudiosos del tema para mejorar el impacto de la movilización del conocimiento en la realidad educativa.

Palabras clave: movilización del conocimiento, conocimientos, universidades, difusión de conocimientos

\begin{abstract}
This article analyzes the concept of knowledge mobilization from its importance as a novel term in the contemporary educational context. The ways in which knowledge is transmitted to the educational community generated the emergence of a growing line of research that analyzes how these knowledges are mobilized from universities and research centers to educational agents and society. Through an exhaustive theoretical review, this paper presents the fundamentals of the concept, describes empirical studies that address it and mentions the suggestions that researchers of the subject themselves made to improve the impact of the mobilization of knowledge in the educational reality.
\end{abstract}

Keywords: knowledge mobilization, knowlegde, universities, dissemination of knowledge

\footnotetext{
${ }^{1}$ Doctora del Programa Oficial de Doctorado en Educación en la Universidad Autónoma de Madrid, España. Máster en gestión educativa (2010) por la Universidad Metropolitana de Ciencias de la Educación, Chile; licenciada en Educación (2007) por la Universidad de La Serena, Chile; profesora de Castellano y Filosofía (2007) por la Universidad de la Serena, Chile. Ha realizado trabajo docente en enseñanza básica, media, preuniversitaria y universitaria en entidades chilenas. Se ha desempeñado como editora de evaluaciones para consultoras educacionales y ha participado como formadora de docentes en el área de competencias lingüísticas de la pedagogía. Ha evaluado revistas de investigación españolas y su trabajo investigativo se centra en la movilización de los conocimientos, en la justicia social y en la relación entre investigación y práctica docente.
} 


\section{INTRODUCCIÓN}

En la educación se producen, día a día y en distintas latitudes, un sinfín de estudios e investigaciones que dan cuenta de nuevos conocimientos. Algunos desarrollan y fundamentan una teoría, otros generan resultados empíricos, pero, si algo tienen en común, es que todos buscan un objetivo similar: comunicar una aportación inédita a la comunidad científica y a la sociedad en su conjunto. La forma en que este conocimiento es transmitido despierta una serie de opiniones y conjeturas, principalmente por el escepticismo frente a la manera en que llega a la comunidad: ¿existe una real difusión de los saberes producidos al interior de las universidades o centros de investigación?

Es en este punto donde surge el concepto de movilización del conocimiento (knowledge mobilization), término que - abreviado como KM por sus iniciales en inglésintenta superar el modelo de monopolio intelectual donde los saberes permanecen en un plano alejado de los posibles usuarios (Levin, 2011). El término movilización del conocimiento fue acuñado en el 2004 por el Consejo de Investigación de Ciencias Sociales y Humanidades de Canadá (SSHRC) para referirse a los esfuerzos por superar la distancia entre la producción de conocimientos, sus repercusiones prácticas y la política.

Con el argumento de que gobiernos, universidades y sistemas educativos están buscando nuevas formas de compartir los conocimientos, el KM incluye los esfuerzos intencionales para incrementar el uso de evidencias que estén basadas en datos recogidos a través de procesos empíricos (Cooper, Levin y Campbell, 2009). Lo que se pretende es que los hallazgos de las investigaciones se transfieran a la sociedad a través de mecanismos planificados que puedan prolongarse en el tiempo.

Si bien el objetivo central de la movilización del conocimiento es fortalecer las relaciones entre la investigación, la política y la práctica, su puesta en funcionamiento en el ámbito educativo no está exenta de dificultades. En la actualidad prevalece la dinámica de relaciones jerárquicas donde los posibles usuarios de los conocimientos reciben de manera pasiva la información. En algunos de los casos, los agentes educativos ni siquiera la reciben, ya que no es un contenido al que se puede acceder con facilidad. Además, existe un conocimiento limitado o deficiente sobre qué es el KM y sobre cuáles son sus objetivos. Esto supone un problema al interior de las organizaciones, ya que, si las personas no conocen en profundidad la temática, difícilmente podrán dimensionar su importancia.

El autor canadiense Ben Levin (2008) explicita algunas interrogantes en torno al $\mathrm{KM}$ que pueden plantearse las organizaciones, los investigadores, los políticos y los educadores, como punto de partida para comenzar a trabajar sobre él: 
- ¿Qué hay que hacer para mejorar el conocimiento sobre el KM?, ¿qué investigaciones, herramientas, prácticas y protocolos deben ser desarrollados? ¿Qué tipos de datos son necesarios?

- ¿Qué tipo de esfuerzos para promover la movilización del conocimiento funcionan para qué circunstancias?

- ¿Cuáles son los beneficios institucionales del desarrollo del KM?, ¿cuáles son las principales dificultades que enfrenta?

- ¿Qué tipos de infraestructura se necesitan para apoyar más eficazmente al KM?, ¿qué tipos de capacidades, sistemas, recursos y relaciones deben ser desarrolladas?

Estas interrogantes, al no estar completamente resueltas, se convierten en el punto de partida de los investigadores que se han interesado en el KM en educación (Arsenijević, 2011; Cooper, 2010; Cooper y Levin, 2010; Cooper et al., 2009; Cooper, Rodway-Macri y Read, 2011; Gaudet, 2013; Levin, 2004, 2008, 2011, 2013; Levin y O'Donnell, 1999; Moss, 2013; Qi y Levin, 2013; Sá, Li y Faubert, 2011).

Si bien es un concepto en proceso de construcción, y relativamente actual en el ámbito educativo, se encuentran disponibles algunas investigaciones con datos empíricos sobre el KM, que resulta importante explicitar. Se describen a continuación cuatro estudios que lo abordan.

En uno de esos trabajos, Amanda Cooper y sus colaboradores (2011) se propusieron conocer las prácticas de movilización del conocimiento que llevaban a cabo los investigadores y un grupo de líderes educativos. Para ello, realizaron un estudio con un enfoque mixto, centrado en once facultades de educación. En una primera fase, realizaron entrevistas telefónicas semiestructuradas a quince decanos y dos vicedecanos, y en una segunda etapa aplicaron encuestas a 278 investigadores de dichas facultades.

Los resultados de ese estudio evidencian que las estrategias de movilización del conocimiento no están integradas en el ámbito organizacional en las facultades, y que las iniciativas que sí se producen, como la publicación de estudios en revistas académicas o la asistencia a conferencias, no son consideradas como elementos importantes para el desarrollo del KM, ya que se las valora como simples actividades de rutina.

Los participantes del estudio coinciden al referirse a las barreras que enfrenta el $\mathrm{KM}$ al interior de las facultades: limitaciones de dinero y tiempo, dificultad para establecer objetivos realizables, problemas en la difusión de la investigación académica al público 
en general, inconvenientes en la coordinación institucional y falta de un liderazgo estable y comprometido con el tema.

Además de estos elementos, existen otros dos factores que obstruyen los canales de la movilización del conocimiento: la falta de historia del KM en las ciencias sociales, y las actitudes divididas sobre su importancia entre los miembros de las facultades. La superación de estos dos factores supone un gran desafío para la organización, ya que si los miembros de la institución no conocen qué es y para qué sirve la movilización del conocimiento, no entenderán el sentido de trabajarla y potenciarla.

Entre las conclusiones del citado trabajo se indica que los investigadores ocupan la mayor parte de su jornada en la realización de investigaciones, publicaciones y eventos académicos, y dedican muy poco tiempo a las redes de difusión de sus trabajos en la comunidad educativa. Esto puede explicarse por la estructura que tiene el círculo académico de la investigación, donde los investigadores deben buscar rápidamente financiación para culminar sus proyectos y comenzar otros, en lugar de dedicar tiempo a hacer un esfuerzo por trabajar en la transferencia y en el impacto de los resultados que producen.

Creso M. Sá y sus colegas (2011), por su parte, llevaron a cabo un estudio cualitativo para conocer cómo las facultades de educación estaban potenciando la movilización del conocimiento. La investigación se centró en trece facultades (cinco en universidades de Estados Unidos, una en Singapur, cinco en Canadá, una en Inglaterra y una en Australia) donde los investigadores realizaron entrevistas telefónicas a 300 líderes, entre rectores, decanos y vicedecanos.

Los resultados de ese estudio aportan puntos de vista positivos y expectativas optimistas sobre la movilización del conocimiento, ya que los líderes lo consideran un tema valioso y que es necesario trabajar en sus instituciones. A pesar de esta valoración positiva, solo en dos de las trece facultades los líderes informan que cuentan con apoyo institucional para fomentar las relaciones con los usuarios potenciales de la investigación, y que implementan este tipo de trabajo de manera explícita. Los líderes de la mayoría de los centros educativos afirman que la movilización del conocimiento sí se produce en sus instituciones, pero de manera irregular y desorganizada. Se destacan múltiples barreras que impiden mayores esfuerzos: las limitaciones financieras, la falta de orientación frente a las fuentes de información, las dificultades para coordinar las iniciativas y los problemas para definir objetivos mensurables.

El estudio concluye que, frente a estas dificultades, debe haber mayor atención a los niveles organizacionales inferiores e intermedios de las instituciones, de modo de descentralizar el trabajo que se realiza en las facultades. La idea es que todos los integrantes de las instituciones sean partícipes de los procesos que allí se llevan a cabo y no se sientan espectadores que reciben información desde un núcleo central inaccesible. 
Lejos de los contextos mencionados en los estudios anteriores, se encuentra una investigación realizada en Serbia en el año 2011. En un trabajo pionero en su país, Jasmina Arsenijević (2011) se propuso evaluar la presencia del KM en el ámbito educativo de las facultades de educación, mediante entrevistas a 90 profesores y a 369 estudiantes.

Los resultados de dicho estudio indican que hay un interés considerable entre los docentes por ampliar sus conocimientos, pero desde una perspectiva personal y no desde la creación de conocimientos en lo colectivo. La generalidad de los participantes proporciona una mirada individualista del conocimiento, que predomina en la sociedad actual, y considera que es una situación que obstaculiza la movilización de los conocimientos.

Tanto docentes como estudiantes coinciden en que el proceso del KM está mayormente en las aulas y, en menor medida, entre el personal docente. Se debaten y se discuten los saberes en niveles específicos y no a través de redes de interacción más globales. Además, la cultura del KM es reconocida como un proceso de adquisición de conocimientos y no como un asunto de distribución o difusión de estos. El estudio concluye que, mientras no exista una cultura de interacción al interior de las instituciones, gestionada por cada administración, el KM seguirá manifestándose solo a través de esfuerzos individuales que no logran trascender hacia políticas generalizadas de transferencia.

Finalmente, en el año 2013, Qi y Levin (2013) buscaron conocer y valorar los esfuerzos que realizaban algunas organizaciones para difundir la investigación a través de sus sitios web, por lo que analizaron los resultados de 100 instituciones. Su trabajo surgió de la premisa de que la comunicación de la investigación había sido transformada a través del desarrollo de las páginas web, ya que, para muchas personas, los sitios online son la principal fuente para conocer estudios o leer artículos de investigación.

Los investigadores comenzaron su estudio observando las actividades de difusión de la investigación en los sitios web de un gran número de organizaciones, mediante una búsqueda deliberadamente amplia con universidades, ministerios de educación y distritos escolares. Los análisis fueron realizados por un equipo de estudiantes de posgrado y profesores, con el objetivo de desarrollar un conjunto común de criterios para la evaluación de los sitios web a través de una escala Likert. En este trabajo, merece la pena precisar que la importancia de los sitios web al interior de las organizaciones puede comprenderse dentro del concepto de gestión de la información. Sin embargo, la gestión del conocimiento que subyace al concepto de KM involucra muchos más procesos, ya que pretende cubrir las necesidades de creación, organización y difusión de los conocimientos tanto al interior como al exterior de la institución, a través de saberes implícitos y explícitos. Si bien existe una estrecha relación entre los dos procesos, es posible que la gestión de la información se englobe dentro de la gestión del conocimiento (Nonaka, Sasaki y Ahmed, 2001). 
La revisión de cada sitio web se fundamentó en el análisis de la relación entre los tres enfoques principales para la movilización del conocimiento: productos, eventos y redes, así como de los indicadores de calidad para cada enfoque: la facilidad de uso, la accesibilidad y el enfoque de audiencia.

En general, los resultados del estudio muestran que son escasos los esfuerzos que realizan estas organizaciones por compartir la investigación, y que la mayoría de las iniciativas de difusión que sí existen se manifiestan unidireccionalmente desde quienes producen la información hacia los potenciales usuarios. Las instituciones mejor evaluadas en sus acciones son aquellas en las que existen algunas actividades de difusión y promoción de los conocimientos a través del sitio online. Sin embargo, no es lo que ocurre en la generalidad de las organizaciones analizadas.

Otro de los resultados más relevantes es que solo un $30 \%$ de los sitios web tienen una clara declaración de sus intenciones frente a la movilización del conocimiento. Además, el $54 \%$ de las organizaciones no tienen espacio para evaluaciones 0 comentarios en el sitio web, frente a un $6 \%$ que sí cuenta con ello. Solo el $38 \%$ de los sitios ofrecen la descarga gratuita de todos sus estudios, mientras que el $29 \%$ no publicita eventos relacionados con la investigación, y menos de la mitad de los que sí lo hacen comentan luego qué ha sucedido con estos eventos. Por último, alrededor del $65 \%$ de los sitios pertenecen a algún tipo de red más global relacionada con la investigación, pero en la mayoría eso no se explicita claramente, ni tampoco se proporciona un acceso directo a las actividades de la red.

Una de las reflexiones finales del estudio es que, si la investigación busca ayudar a mejorar la educación, debe tomar medidas más activas para que los conocimientos favorezcan los cambios en la práctica. Los autores asumen que los esfuerzos actuales no han sido suficientes, por lo que sugieren que compartir conocimientos derivados de la investigación debe convertirse en un tema prioritario y no opcional para las organizaciones que los producen.

\section{Sugerencias del KM para disminuir la distancia entre la investigación}

\section{y la práctica educativa}

Algunos de los autores que trabajan sobre la movilización del conocimiento realizan una serie de sugerencias para lograr que los conocimientos estén más cerca de la práctica educativa, impacten en ella y disminuyan la brecha histórica entre ambos conceptos (Cooper, 2012; Cooper y Levin, 2010; Cooper et al., 2009; Fenwick y Farrell, 2011; Gaudet, 2013; Levin, 2004, 2011, 2013; Moss, 2013; Qi y Levin, 2013; Sá et al., 2011). Estas sugerencias se enfocan principalmente en dos elementos. Por una parte, en la interacción entre la producción y el uso del conocimiento, como aspectos que deben modificar sus formas tradicionales de comunicación. Y, por otra parte, en la importancia 
de los intermediarios, como las personas que actúan de vínculo entre el conocimiento científico y la práctica.

\section{Producción, uso e interacción del conocimiento}

Para superar las barreras entre el conocimiento y la práctica es necesario focalizar la atención en los procesos de organización, de acuerdo al contexto educativo desde donde emergen, sobre el entendido de que la movilización del conocimiento depende en gran medida de las interacciones entre productores y usuarios dentro de escenarios reales como, por ejemplo, las aulas o las propias universidades (Levin, 2011).

Los esfuerzos del KM para acercarse a la práctica educativa deben enfocarse principalmente en tres elementos: la producción de la investigación, el uso de ella y la vinculación entre la producción y el uso (Cooper, 2012; Fenwick y Farrell, 2011; Levin, 2004, 2011).

Figura N. ${ }^{\circ} 1$

Movilización del conocimiento en educación

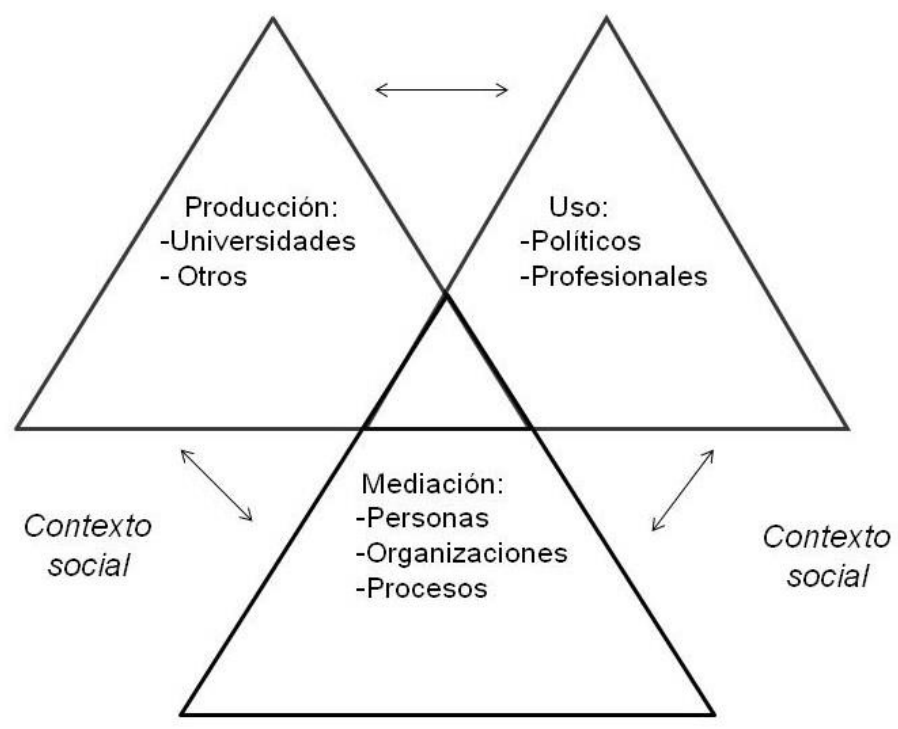

Fuente: Mobilizing Research Knowledge in Education (Levin, 2011, p.16).

Para optimizar la presencia del KM en educación, en primer lugar, se debería mejorar la producción de conocimientos. Para lograrlo, Levin (2004) propone las siguientes ideas:

- Prestar mayor atención a los criterios que determinan la entrega de recursos, dando importancia a la evaluación de los financiamientos. 
- Proporcionar un listado de sugerencias y posibles estrategias para el impacto, que podría animar a los investigadores a prestar mayor atención a este aspecto de su trabajo.

- Proporcionar, desde las universidades, un apoyo a la movilización de los conocimientos en educación similar al que entregan a las ciencias o a la ingeniería.

- Fortalecer la entrada de los usuarios potenciales en el desarrollo y revisión de las investigaciones propuestas. Hay muchos casos en los que un debate temprano entre investigadores y usuarios puede dar lugar a estudios más sólidos, así como un mayor interés por parte de los usuarios potenciales.

- Adoptar medidas para extraer el máximo provecho de la investigación existente. A menudo, los investigadores se involucran en nuevos proyectos antes de aprovechar completamente el potencial del último que hicieron. Sería beneficioso alentar a los investigadores a dedicar más tiempo a la explotación de sus datos en lugar de ir inmediatamente a un nuevo estudio.

- Construir redes de investigadores y usuarios con intereses comunes como una manera de desarrollar programas de investigación a mayor escala y vínculos con otras iniciativas.

En segundo lugar, se debe mejorar el uso de los conocimientos adquiridos, al considerar que estos no tendrán un real impacto si sus potenciales beneficiarios no están interesados en utilizarlos. Es de vital importancia que los agentes educativos encuentren, comprendan y utilicen los saberes; sin embargo, poco se sabe sobre cómo lograrlo (Cooper et al., 2009). Es necesario trabajar con las principales organizaciones de usuarios, y aprender más sobre sus capacidades y limitaciones. Para lograrlo, se hace indispensable impulsar iniciativas concretas, como la creación de redes de usuarios, prácticas de intercambio de conocimientos basados en Internet o aumentar la dotación de personal con buenos antecedentes en investigación.

También se deben desarrollar mejores medios de intercambio de información entre las organizaciones escolares, que incluyan procesos compartidos de revisión de la literatura, mejoras en los medios de intercambio de información sobre investigación relevante, y la creación de redes de funcionarios de distintas organizaciones que compartan responsabilidades en la difusión de la investigación. 
Finalmente, se deben establecer vínculos entre la producción y el uso, a través de una serie de medidas concretas. Una de ellas es la "traducción" de los resultados de la investigación, con el objetivo de superar las barreras del lenguaje científico propio de las investigaciones. Dichos resultados, además de ser explicados con claridad a los agentes educativos, deben ser difundidos a través de vehículos comunicacionales tecnológicos y también interpersonales.

Los recursos que ofrece Internet en la actualidad son poco aprovechados por las organizaciones y por los investigadores. Las páginas web, los servicios de mensajería instantánea y el material audiovisual que la tecnología permite realizar no han sido utilizados de forma adecuada para atraer la atención de los potenciales usuarios del conocimiento. Aprovechar mejor estos recursos puede concretarse, por ejemplo, con la creación de sitios web más llamativos, con enlaces directos a investigaciones de interés o con videos que expliquen visualmente los resultados de un estudio (Gaudet, 2013; Levin, 2008; Qi y Levin, 2013).

Aunque la ayuda de la informática es importante, no debe olvidarse que el intercambio eficaz entre los investigadores y los agentes educativos también requiere del contacto cara a cara entre las personas, que sigue siendo trascendental para lograr confianza e interés entre ellas. Por lo tanto, lo que se necesita es un equilibrio entre el uso de los recursos de la tecnología y las instancias de diálogo donde los sujetos expresan sus opiniones, inquietudes y pensamientos.

Por último, mejorar los vínculos entre la producción y el uso del conocimiento requiere construir conexiones de comunicación más precisas. Esto puede lograrse con la asignación de personas que posean competencias y habilidades propias del KM y que desarrollen iniciativas donde todo el personal se sienta partícipe: la participación de los intermediarios.

\section{Participación activa de los intermediarios}

Existen muchos términos que describen la participación de mediadores dentro de los procesos, por ejemplo, agentes de conocimiento, agencias de terceros o facilitadores (Honig, 2004; Levin, 2008; Thompson, Estabrooks y Degner, 2006; Ward, House y Hamer, 2009; Ward, Smith, Foy, House y Hamer, 2010). Con independencia de las distintas expresiones que se utilizan para referirse a los intermediarios, en términos generales, estos han sido entendidos como los elementos que actúan entre dos o más espacios (Honig, 2004), cuya principal función es mediar o gestionar que existan cambios en las partes involucradas. Los intermediarios buscan proporcionar un valor diferente, que vaya más allá de aquello que las partes, por sí solas, serían capaces de desarrollar. Sin embargo, al mismo tiempo que buscan diferenciarse de los espacios en los que intervienen, dependen de ellos para llevar a cabo sus funciones esenciales (Feldman, Nadash y Gursen, 2001). 
En la movilización del conocimiento en educación, los intermediarios son entendidos como personas encargadas de acercar los conocimientos a la práctica al interior de las organizaciones - por ejemplo, universidades o centros escolares_, lo que no es una tarea fácil. Sucede que los intermediarios funcionan principalmente en el espacio "en blanco" de los sistemas, lo que complejiza su visibilidad (Cooper, 2012). Aunque gran parte de las interacciones y funciones de las instituciones ocurren en estos espacios, a menudo nadie se hace responsable de ellos.

El desafío de las organizaciones es otorgar mayor importancia al rol de los intermediarios a través de acciones concretas como, por ejemplo, invertir en su formación. Se les debe preparar de forma adecuada, ya que no se trata de elegir aleatoriamente a personas de la organización sin preparación en materias del KM. Además, se les deben explicitar sus funciones y responsabilidades, al mismo tiempo que se les facilitan los recursos personales y económicos que requiere su trabajo (Levin, 2008). Teniendo la preparación correspondiente, claridad sobre sus objetivos y los recursos que necesitan, los intermediarios cuentan con las condiciones necesarias para llevar a cabo acciones concretas. Estas acciones, por mencionar algunas, pueden ser la traducción del lenguaje científico a uno menos especializado y la formulación de acciones de interacción que sean atractivas para los investigadores y para los agentes educativos. Lograr que estas acciones despierten el interés de los involucrados requiere que los intermediarios movilicen actividades creativas e innovadoras, que rompan con las modalidades dialógicas típicas.

Amanda Cooper (2010), profesora de la Universidad de Queen (Canadá), realiza una propuesta de cuatro pasos a través de los cuales los intermediarios pueden fomentar la movilización del conocimiento al interior de las organizaciones educativas. En ellas, los intermediarios:

1) Identifican el problema que enfrenta la institución: ¿qué tipos de brechas existen entre la investigación y la práctica? Detectar el tipo de brecha entre la investigación, la política y la práctica es esencial para el KM, ya que, en gran medida, la delimitación del problema determina qué tipo de estrategias y esfuerzos son necesarios para mejorar la situación.

2) Analizan sus funciones específicas en los problemas detectados, y determinan qué acciones personales llevarán a cabo y qué recursos solicitarán a la institución.

3) Determinan las estrategias a utilizar, dependiendo del problema de KM que se está tratando de resolver. Pueden establecer instancias de interacción, reuniones interdepartamentales, seminarios de investigación, etcétera, dependiendo de los problemas principales que identificaron en el paso 1. 
4) Crean un plan estratégico de KM capaz de adaptarse al contexto organizacional, el que difunden en la organización explicitando las metas que pretenden lograr.

\section{CONCLUSIONES}

Un primer paso para que el KM logre ingresar y permanecer en el mundo educativo es la concientización acerca del tema, lo que incluye, necesariamente, que se expliciten sus objetivos e implicancias. Para que esto sea posible, los investigadores educativos deben recibir una formación específica en torno a la importancia de la movilización del conocimiento, que vaya más allá de conocer el significado literal del concepto. Quienes investigan los problemas educativos deben asumir una responsabilidad casi inherente a su profesión, que no es otra que difundir aquello que descubren.

En este punto es interesante mencionar algunas aportaciones que dan cuenta del conocimiento tácito e implícito que se produce al interior de las organizaciones, como parte de una reflexión acerca de las dificultades que enfrenta el KM. Los conocimientos tácitos tienen un tremendo valor intrínseco, pero al mismo tiempo son los más difíciles de gestionar. Al respecto, Nonaka y Takeuchi (1995) indican que la manera en que el conocimiento se crea es a partir de la conversión del conocimiento tácito en conocimiento explícito. El conocimiento tácito tiene, a su vez, dos dimensiones: una técnica (el knowhow), centrada en cómo llevar a cabo un trabajo, y una cognoscitiva, que alude a la imagen que cada persona posee sobre la realidad. Para Carla O'Dell (2001), el conocimiento tácito es el know-how, lo que incluye a su vez la intuición y el razonamiento que da la experiencia.

En esta misma línea, Davenport y Prusak (2001) mencionan que el conocimiento al interior de las organizaciones se expresa no solo por medio de documentos tangibles, sino también en las rutinas, procesos, prácticas y normas institucionales. Con esto, queda claro que el conocimiento no es algo ordenado o simple, sino que también es intuitivo y, por lo tanto, difícil de explicitar en palabras o de entender completamente en términos tradicionales.

El conocimiento no puede permanecer en un ámbito de élite, abstracto y alejado de la realidad, porque en este caso de nada sirve invertir tiempo y recursos en producirlo. Si no existe una conexión entre los conocimientos y la práctica no tiene mayor sentido investigar y publicar. 
Una de las dificultades que atraviesa el KM tiene que ver con los criterios exigidos a los investigadores para la publicación de sus trabajos. En el contexto español, el uso del Journal Citation Report (JCR) es tan profundo ${ }^{2}$ que condiciona y justifica la vida de muchos investigadores: según los artículos publicados en revistas indexadas en el JCR lograrán o no acreditarse y, con ello, conseguir un trabajo en la Universidad.

En consecuencia, los docentes universitarios se esmeran en publicar artículos JCR, que les den reconocimiento y prestigio, lo que incide en la forma en que observan la movilización de los conocimientos. Si el investigador busca publicar en revistas JCR focalizará su trabajo en dicho objetivo, por lo que impactar en la práctica educativa o ser leídos por directores o profesores queda relegado a un plano secundario. Probablemente los investigadores sí tienen la buena intención de escribir artículos que tengan una utilidad en la práctica, pero también son conscientes de que su actividad investigadora está siendo evaluada de acuerdo a ciertos parámetros.

Superar estas dificultades requiere que las administraciones tengan criterios diferenciadores para evaluar la investigación educativa, donde se valoren positivamente otras iniciativas de comunicación de la investigación además de las revistas JCR. También es importante que surjan cambios al interior de las universidades, las que deben promover nuevas políticas de difusión de los saberes producidos. Un ejemplo de ello es la optimización de las páginas web, tanto en el plano institucional como también en el ámbito más específico de cada facultad. Si bien estas plataformas tienen más bien un objetivo informativo en cuanto a plazos, asignaturas y organigrama, pueden hacer el intento de visibilizar con mayor claridad y accesibilidad los estudios, artículos y publicaciones que han surgido en su interior.

La importancia de la temática abordada en este artículo no es menor: si los conocimientos que producen las investigaciones no logran movilizarse, ¿para qué se investiga? Si lo que mueve la investigación son los sexenios y acreditaciones, hay algo que queda pendiente. Se ha pasado de querer cambiar el mundo con la investigación educativa a conformarse con recopilar papeles para un hipotético ascenso laboral. Los investigadores se leen unos a otros, se citan entre ellos, pero terminan escribiendo para una academia reducida y exclusiva que nada tiene que ver con la realidad.

Las nuevas líneas de investigación que pueden surgir a partir de este estudio son, sin duda, la indagación empírica de esta realidad al interior de las universidades o centros de investigación. Se puede analizar el funcionamiento comunicacional de las facultades, las formas de difusión de cada institución, la presencia o ausencia de los intermediarios, el impacto de las páginas web, entre otros temas.

Si el trabajo de los investigadores no se moviliza, no sale de su espacio de producción y no es capaz de llegar a los agentes educativos y a la sociedad en general,

\footnotetext{
${ }^{2}$ En investigación educativa en España, en los últimos años, se están empezando a considerar los artículos publicados en Scopus (o SJR - Scimago Journal Rank) tanto para la evaluación positiva de los tramos de investigación como para las acreditaciones, pero ese hecho no altera la lógica de la supeditación de la investigación a parámetros no siempre objetivos impuestos por una empresa privada.
} 
estos no merecen llamarse investigadores educativos. Todos los hallazgos deben tener como fin último impactar en la práctica, generar cambios sustanciales, despertar acciones innovadoras y superar los paradigmas establecidos.

\section{REFERENCIAS}

Arsenijević, J. (2001). Methodology for assessment of knowledge management in higher education institutions. African Journal of Business Management, 5(8), 3168-3178.

Cooper, A. (octubre, 2010). Knowledge mobilization intermediaries in education. Comunicación presentada en la $39^{a}$ Annual Canadian Society for the Study of Education Conference, Montreal, Canadá.

Cooper, A. (2012). Knowledge mobilization intermediaries in education: A cross-case analysis of 44 Canadian organizations (Tesis doctoral). Universidad de Toronto, Toronto, Canadá.

Cooper, A., y Levin, B. (2010). Some Canadian contributions to understanding knowledge mobilization. Evidence \& Policy: A Journal of Research, Debate and Practice, 6(3), 351-369.

Cooper, A., Levin, B., y Campbell, C. (2009). The growing (but still limited) importance of evidence in education policy and practice. Journal of Educational Change, 10(2-3), 159-171.

Cooper, A., Rodway-Macri, J., y Read, R. (setiembre, 2011). Knowledge mobilization practices of educational researchers in Canada. Comunicación presentada en el American Educational Research Association, New Orleans, Estados Unidos.

Davenport, T., y Prusak, L. (2001). Conocimiento en acción como las organizaciones manejan lo que saben. Buenos Aires, Argentina: Pearson Education.

Feldman, P., Nadash, P., y Gursen, M. (2001). Improving communication between researchers and policy makers in long-term care or, researchers are from Mars; policy makers are from Venus. The Gerontologist, 4(3), 312-321.

Fenwick, T., y Farrell, L. (2011). Knowledge mobilization and educational research: politics, languages and responsibilities. Nueva York, NY: Routledge.

Gaudet, J. (2013). It takes two to tango: knowledge mobilization and ignorance mobilization in science research and innovation. Prometheus, 31(3), 169-187.

Honig, M.I. (2004). The new middle management: Intermediary organizations in education policy implementation. Educational Evaluation and Policy Analysis, 26(1), 65-87.

Levin, B. (2004). Making research matter more. Education Policy Analysis Archives, 12(56), 1-20.

Levin, B. (mayo, 2008). Thinking about knowledge mobilization. Paper presentado en el Canadian Council on Learning y The Social Sciences and Humanities Research Council, Toronto, Canadá.

Levin, B. (2011). Mobilizing research knowledge in education. London Review of Education, 9(1), 15-26.

Levin, B. (2013). To know is not enough: Research knowledge and its use. Review of Education, 1(1) 2-31.

Levin, J., y O'Donnell, A. (1999). What to do about educational research's credibility gaps? Issues in Education 5(2), 177-229.

Moss, G. (2013). Research, policy and knowledge flows in education: what counts in knowledge mobilization? Contemporary Social Science, 8(3), 237-248. 
Nonaka, I., Sasaki, K., y Ahmed, M. (2001). Knowlogde creation. The power of tacit knowlodge. Knowledge Management Magazine, 10, 10-15.

Nonaka, I., y Takeuchi, H. (1995). The Knowledge-creating company. How japanese companies create the dynamics of innovation. New York, NY: Oxford University Press.

O'Dell, C. (2001). El valor de la gestión del conocimiento. Harvard Deusto Márketing y Ventas, 46, 18-20.

Qi, J., y Levin, B. (2013). Assessing Organizational Efforts to Mobilize Research Knowledge in Education. Education Policy Analysis Archives, 2(2), 18-31.

Sá, C., Li, S., y Faubert, B. (2011). Faculties of education and institutional strategies for knowledge mobilization: an exploratory study. Higher Education, 61(4), 501-512.

Thompson, G., Estabrooks, C., y Degner, L. (2006). Clarifying the concepts in knowledge transfer: a literature review. Journal of Advanced Nursing, 53(6), 691-701.

Ward, V., House, A., y Hamer, S. (2009). Knowledge brokering: the missing link in the evidence to action chain? Evidence \& Policy: a Journal of Research, Debate and Practice, 5(3), 267-279.

Ward, V., Smith, S., Foy, R., House, A., y Hamer, S. (2010). Planning for knowledge translation: a researcher's guide. Evidence y Policy: A Journal of Research, Debate and Practice, 6(4), 527-541. 